\title{
Excited state absorption spectra of dissolved and aggregated distyrylbenzene: A TD-DFT state and vibronic analysis
}

Cite as: J. Chem. Phys. 147, 034903 (2017); https://doi.org/10.1063/1.4993216

Submitted: 25 April 2017 . Accepted: 26 June 2017 . Published Online: 18 July 2017

Eliezer Fernando Oliveira, Junqing Shi, Francisco Carlos Lavarda, Larry Lüer, Begoña Milián-Medina, and Johannes Gierschner (D)

\section{ARTICLES YOU MAY BE INTERESTED IN}

A consistent and accurate ab initio parametrization of density functional dispersion correction (DFT-D) for the 94 elements $\mathrm{H}-\mathrm{Pu}$

The Journal of Chemical Physics 132, 154104 (2010); https://doi.org/10.1063/1.3382344

Natural transition orbitals

The Journal of Chemical Physics 118, 4775 (2003); https://doi.org/10.1063/1.1558471

Fluorescence and absorption spectra of oligophenylenevinylenes: Vibronic coupling, band shapes, and solvatochromism

The Journal of Chemical Physics 116, 8596 (2002); https://doi.org/10.1063/1.1469612

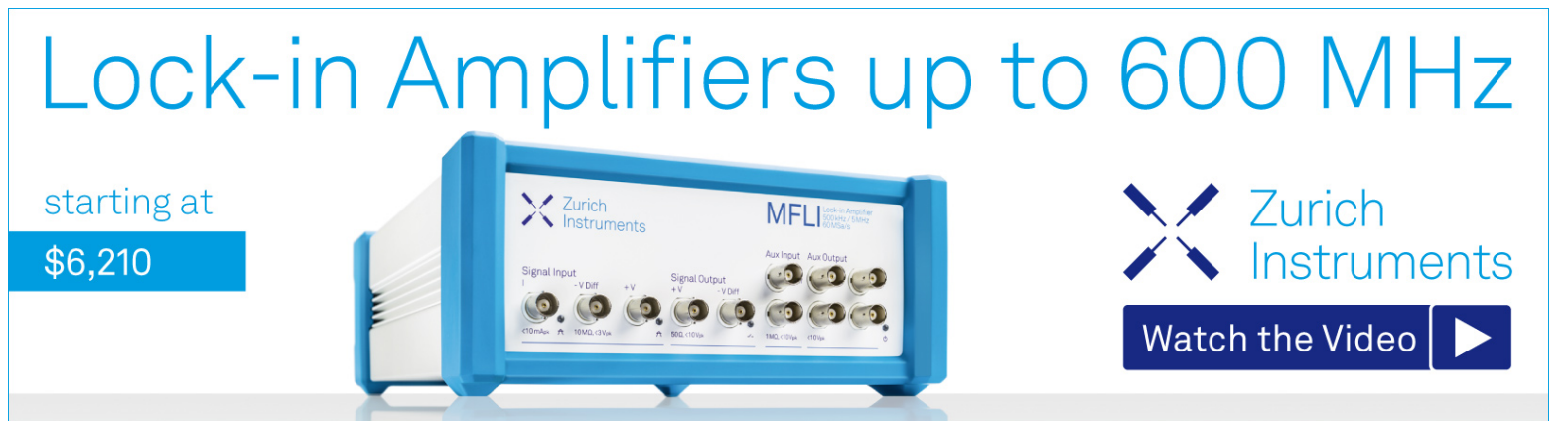




\title{
Excited state absorption spectra of dissolved and aggregated distyrylbenzene: A TD-DFT state and vibronic analysis
}

\author{
Eliezer Fernando Oliveira, ${ }^{1}$ Junqing Shi, ${ }^{2}$ Francisco Carlos Lavarda, ${ }^{1,3}$ Larry Lüer, ${ }^{2}$ \\ Begoña Milián-Medina, 2,4,a) and Johannes Gierschner ${ }^{2, a)}$ \\ ${ }^{1}$ UNESP-Universidade Estadual Paulista, POSMAT-Graduate Program in Material Science and Technology, \\ Av. Eng. Luiz Edmundo Carrijo Coube 14-01, 17033-360 Bauru, SP, Brazil \\ ${ }^{2}$ Institute for Advanced Studies, IMDEA Nanoscience, C/ Faraday 9, Campus Cantoblanco, \\ 28049 Madrid, Spain \\ ${ }^{3}$ DF-FC, UNESP-Universidade Estadual Paulista, Av. Eng. Luiz Edmundo Carrijo Coube 14-01, \\ 17033-360 Bauru, SP, Brazil \\ ${ }^{4}$ Department for Physical Chemistry, Faculty of Chemistry, University of Valencia, Avenida Dr. Moliner 50, \\ 46100 Burjassot, Valencia, Spain
}

(Received 25 April 2017; accepted 26 June 2017; published online 18 July 2017)

\begin{abstract}
A time-dependent density functional theory study is performed to reveal the excited state absorption (ESA) features of distyrylbenzene (DSB), a prototype $\pi$-conjugated organic oligomer. Starting with a didactic insight to ESA based on simple molecular orbital and configuration considerations, the performance of various density functional theory functionals is tested to reveal the full vibronic ESA features of DSB at short and long probe delay times. Published by AIP Publishing. [http://dx.doi.org/10.1063/1.4993216]
\end{abstract}

\section{INTRODUCTION}

Over the past 20 years, pump-probe spectroscopy (PPS) has evolved as an exceedingly powerful tool to analyze the photophysics in complex systems, in particular of natural and artificial light harvesting as well as of organic optoelectronics, whose functionalities are all based on aggregated conjugated organic materials. ${ }^{1-4}$ Meanwhile, PPS is becoming a widespread turn-key like technique, which covers the UV to IR spectral range with widely tunable pump wavelength, timeresolution from milliseconds (ms) down to femtoseconds (fs), and high sensitivity, and matrix-based data analysis methods ${ }^{5}$ have, in some cases, allowed the complete analysis of elementary transfer processes by identifying single species PPS spectra and their evolution in time. . $^{3,4,6,7}$ The sensitivity of the technique has been pushed far enough so that PPS is now routinely used for the detection of elementary loss channel in lasing materials ${ }^{8}$ and organic solar cells under operational conditions. ${ }^{6,9,10}$

One general difficulty in the analysis of PPS is spectral congestion caused by a multitude of optical probes from various photoexcited states such as singlet, triplet, and charged states. In order to quantify the photoexcitation dynamics, these optical probes often need to be obtained by additional, dedicated experiments (such as chemically sensitizing triplet states ${ }^{11}$ or electrically generating charged states in the sample under consideration ${ }^{12}$ ). These additional necessary experiments make the whole procedure time-consuming and tedious, reducing the usefulness of PPS in the optimization of optoelectronic materials and devices. Here, quantum chemistry could provide useful input. This concerns band

\footnotetext{
a) Authors to whom correspondence should be addressed: milian@uv.es and johannes.gierschner@imdea.org
}

positions, spectral shapes (number of expected bands and vibronic coupling), and overall oscillator strengths (to obtain concentrations from time-resolved spectra via Lambert-Beer's law).

Quantum-chemical calculations of conjugated molecules (and, to some degree, even of their assemblies), i.e., of ground and excited state geometries and vibrations, as well as of ground state absorption (GSA) and photoluminescence (PL) electronic transitions along with their vibronic couplings are done nowadays with good to reasonable accuracy prior to or accompanying the experimental studies. ${ }^{13-16}$ On the other side, the calculation of excited state absorption (ESA) spectra is much less explored. ${ }^{17}$ This is partly due to the fact that the respective algorithms (based on quadratic response (QR) functions) have been developed merely within a limited number of quantum-chemical methodologies, and have been implemented only in a few quantum-chemical program packages that require some expert knowledge. For these reasons, the number of computational ESA studies on conjugated molecules is quite limited, ${ }^{18,19}$ while the application of QR towards the calculation of two-photon absorption (TPA) is more frequently found in the literature. ${ }^{20-22}$ Furthermore, didactic approaches to understand the constituting factors of the excitations are rather missing; methodology tests are scarce, and more advanced studies, i.e., on vibronic coupling in ESA spectra or the treatment of molecular assemblies have not been carried out until now.

It is the motivation of the present study to fill this gap. For this we have selected a medium-sized molecule, distyrylbenzene (DSB, see Fig. 1), being one of the working horses of optical spectroscopy. In fact, both GSA and PL spectra, ${ }^{23}$ as well as ESA spectra at different probe delay times are fully known from experiment in solution ${ }^{24,25}$ and in the solid state. ${ }^{26,27}$ GSA and PL spectral positions of DSB were 


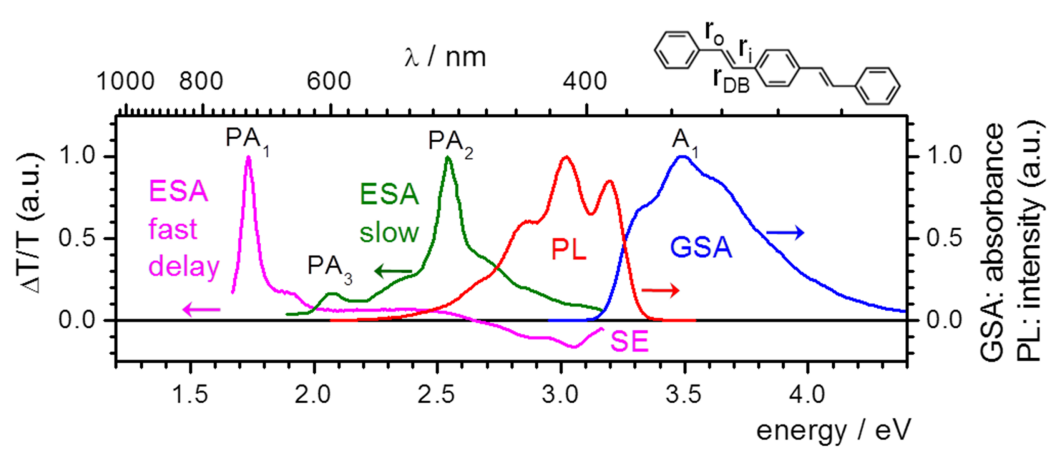

FIG. 1. Experimental spectra of DSB in solution. Red line: PL (in dioxane). Blue line: GSA (in dioxane). Green line: ESA at $1.1 \mu$ s delay time (in toluene by sensitization with benzophenone, $355 \mathrm{~nm}){ }^{25}$ Magenta line: ESA at 0.4 ps delay time (in dioxane; see Sec. II for details). well reproduced by the time-dependent density functional theory (TD-DFT), ${ }^{28,29}$ and vibronic coupling was investigated in an atomistic manner in fluid and solid solutions (including temperature effects ${ }^{30}$ as well as in crystals. ${ }^{31}$ We will therefore calculate GSA and ESA spectra of DSB at the TD-DFT level of theory, providing a reasonable compromise between accuracy and cost efficiency for medium size molecules. In any case, the number of functionals available for ESA calculations in the program package used (Dalton) is limited, so we decided for the BHLYP functional, which performs reasonably well for the calculation of allowed GSA transitions for different types of conjugated molecules of different sizes, ${ }^{13}$ B3LYP and its long-range corrected variant CAMB3LYP were used for comparison (the latter is known to perform similarly well as BHLYP for the calculation of transition between the ground and first excited singlet state $\mathrm{S}_{0}$ $\left.\rightarrow \mathrm{S}_{1}\right){ }^{13}$

We will begin with a didactic insight to $S_{1} \rightarrow S_{n}$ ESA to understand the rather simple structure of the experimental spectrum in solution (Fig. 1); for this we will start from the molecular orbitals (MOs) and mono-electronic configurations, to demonstrate the mandatory need for configuration interaction $(\mathrm{CI})$ in the proper calculation of ESA; this makes ESA more sensitive to the methodology compared with the $\mathrm{S}_{0}$ $\rightarrow \mathrm{S}_{1}$ GSA of DSB. We will therefore test the performance of the various TD-DFT functionals for $S_{1} \rightarrow S_{n}$ and show that BHLYP (and CAM-B3LYP) works reasonably well for this purpose, allowing for a proper state assignment in the experimental ESA spectra. We will then assign $\mathrm{T}_{1} \rightarrow \mathrm{T}_{\mathrm{n}}$ transitions and give evidence for the occurrence of $\mathrm{D}_{0} \rightarrow \mathrm{D}_{\mathrm{n}}$ transitions of radical cations. We will further prove that the remarkably small spectral bandwidth of the main S-S ESA peak is indeed a result of small geometrical reorganization, which can be understood from the topologies of the MOs involved. The full calculation of the vibronics allows for a detailed understanding of the complex ESA spectra by the assignment of vibronic side bands. Finally, we will calculate the vertical transitions of a small aggregate of DSB as a model for the solid state, compare this with the experiment, and reveal the electronic character of the relevant ESA transition.

\section{METHODS}

\section{A. Calculations}

Within the TD-DFT methodology, the transition energies (and transition dipoles) of GSA are obtained as the poles and residues of linear response (LR) functions, while ESA are obtained as those of the quadratic response $(\mathrm{QR}) .^{32}$ Different to LR, only few programs have implemented QR in their codes. For this reason, GSA and ESA were analyzed with different programs. All geometry optimizations (ground and excited singlets, triplets, and cationic states geometries) as well as LR calculations were done in GAUSSIAN $09,{ }^{33}$ while ESA calculations were conducted with DALTON 2016, ${ }^{34}$ which was already used earlier for this purpose. ${ }^{18}$ For (TD)DFT calculations of GSA electronic transitions, the results depend largely on the functional employed, while little is known on ESA; for TPA spectra on the other hand, CAM-B3LYP was already suggested earlier. ${ }^{21,22}$ We therefore chose to verify the performance on ESA for a number of popular functionals with different amounts of Hartree-Fock exchange, i.e., B3LYP $(20 \%),{ }^{35,36}$ BHLYP $(50 \%),{ }^{37}$ as well as the long-range corrected CAM-B3LYP functional. ${ }^{38}$ All calculations were done in vacuum, imposing $\mathrm{C}_{2 \mathrm{~h}}$ symmetry, ${ }^{23,39}$ and employing the 6-311G* basis set. In order to analyze the vibronic couplings, the program FCclasses was employed, including Duchinsky rotation in the distorted, displaced harmonic oscillator model ${ }^{40}$ To calculate the room temperature solution spectra from the stick spectra, a Gaussian convolution was used to account for the dynamic and static contributions of solvent polarizability fluctuations, which is of Gaussian shape due to its statistical nature; here a typical half-width at half maximum of $0.1 \mathrm{eV}$ was applied. ${ }^{30,41}$ To account for the thermal population of low frequency (in particular of torsional) modes, which are widely different in $S_{0}$ and $S_{1}$, for floppy molecules as $\mathrm{DSB}$, an exponential convolution $\mathrm{G}(v)=\exp \{-\mathrm{h} v /(\mathrm{D}-1) \mathrm{kT}\}$ was applied to the absorption spectrum with $\mathrm{D}=5.5$, while for the emission, this is of minor importance; for a detailed discussion and rationalization, see Ref. 30. Analysis of GSA, PL, and ESA of DSB aggregates was performed using a herringbone arrangement ( $\mathrm{H}$-aggregation), in which we used the previously DFT-optimized DSB molecules to place them in the intermolecular arrangement found in the X-ray analysis of DSB single crystals. ${ }^{42}$ GSA of dimers was calculated using the BHLYP, CAMB3LYP, and wB97XD functionals. Calculations for a cluster of four DSB molecules were done (BHLYP) to check the size effects on the cluster's optical properties. ESA calculations (BHLYP) were done for the dimers.

\section{B. Experiment}

The fs transient absorption spectra of DSB were (re)measured (in dioxane) to enable quantitative extraction 
of peak areas. The spectra have been obtained with a regeneratively amplified system at $1 \mathrm{kHz}$ repetition rate (ClarkMXR CPA2101). The $775 \mathrm{~nm}$ pulses were split into two parts: one part was frequency doubled using a lithium triborate crystal (pump pulses) and the other part was focused onto a $3 \mathrm{~mm} \mathrm{CaF}_{2}$ plate on a rotating mount, producing a fs white light super continuum from 320 to $760 \mathrm{~nm}$ (probe pulses). The probe pulses were focused onto the sample (about $150 \mu \mathrm{m}$ diameter) and overlapped with the pump pulse (about $500 \mu \mathrm{m}$ diameter), the delay time of which was controlled via a mechanical translation stage and corner cube. After transmission through the sample, the probe pulses were sent to a prism spectrometer (Entwicklungsbüro Stresing $\mathrm{GmbH}$ ) with a Si CCD array (512 pixels). Data acquisition and modeling was done by custom-built Python software, using open source packages Matplotlib, PyQt4, SciPy, and Pyserial, among others.

\section{RESULTS AND DISCUSSIONS}

\section{A. MOs and configurations}

The experimental GSA and PL spectra, as well as the ESA spectra at short $(20 \mathrm{ps})$ and long probe delay times $(1.1 \mu \mathrm{s})^{25}$ of DSB in solution at room temperature are shown in Fig. 1. The GSA spectrum is largely dominated by a broad, somewhat structured band, centered at $3.52 \mathrm{eV}$, which is known to originate from the $\mathrm{S}_{0}\left(1^{1} \mathrm{~A}_{\mathrm{g}}\right) \rightarrow \mathrm{S}_{1}\left(1^{1} \mathrm{~B}_{\mathrm{u}}\right)$ electronic transition, ${ }^{30}$ and which essentially corresponds to the transition from the highest occupied to the lowest unoccupied molecular orbital (HOMO $\rightarrow$ LUMO). The reason for the virtually mono-configurational description and the dominating intensity (oscillator strength $f$ ) of $1^{1} \mathrm{~A}_{\mathrm{g}} \rightarrow 1^{1} \mathrm{~B}_{\mathrm{u}}$ in the GSA spectrum is readily seen in the $\mathrm{MO}$ diagram and the resulting scheme of mono-electronic configurations $\Phi_{i}$ (Fig. 2); here, $\Phi_{1}$, i.e., the HOMO $\rightarrow$ LUMO excitation, is well separated from $\Phi_{\mathrm{i}}$ (i $>1$ ), thus keeping CI very small; ${ }^{43}$ at the same time, the intensity is high due to the delocalized character of HOMO and LUMO. The next configurations $\Phi_{2}$ and $\Phi_{3}$ are symmetry forbidden $\left(\mathrm{A}_{\mathrm{g}}\right)$, giving rise to two electronic states as linear combinations with almost equal coefficients but opposite sign (alternant pairing). All higher configurations $\left(\Phi_{i}, \mathrm{i}>3\right)$ are energetically very close to their neighbors so that $\mathrm{CI}$ is always very strong; this results in high contributions of localized MOs (i.e., HOMO-n, LUMO+n with $n>1$ ) of the resulting states in all cases, being thus rather small in intensity, in all rationalizing the dominance of the $S_{0} \rightarrow S_{1}$ transition in the GSA spectrum.

For the ESA spectrum originating from $\mathrm{S}_{1}$, the electronic situation exhibits consequently a very different character compared with the GSA. All transitions are expected to show strong CI, see Fig. 2; for this reason, a pronounced dependency of the energies (and ordering) of the final states and their intensities on the applied theoretical methodology is anticipated. In any case, the principal features of the ESA spectrum originating from $\mathrm{S}_{1}\left(1^{1} \mathrm{~B}_{\mathrm{u}}\right.$, which essentially corresponds to $\Phi_{1}$; vide supra) are quite obvious from the MOs and configurations in Fig. 2. The first accessible configurations $\Phi_{2}$ and $\Phi_{3}$ are symmetry allowed $\left(\mathrm{A}_{\mathrm{g}}\right)$ and formed by delocalized MOs, i.e., HOMO-1 $\rightarrow$ LUMO, HOMO $\rightarrow$ LUMO+1 and nearly degenerate (Fig. 2). Linear combination $\left(\mathrm{a}_{1} \cdot \Phi_{2} \pm \mathrm{a}_{2} \cdot \Phi_{3}\right)$ results in $\mathrm{S}_{2}\left(2^{1} \mathrm{~A}_{\mathrm{g}}\right)$ and $\mathrm{S}_{3}\left(3^{1} \mathrm{~A}_{\mathrm{g}}\right)$ states, where one $(+)$ has to carry high oscillator strength $f$, while in the other one $(-) f$ has to be small. All higher excitations $\mathrm{S}_{\mathrm{i}}$ (i $>3$ ) involve localized occupied and/or unoccupied MOs, and are thus rather low in intensity, which is, in particular, true for DSB; for these reasons, the singlet ESA spectrum is expected to be dominated by one intense peak as indeed seen in the experimental spectrum at short pump-probe delay times.

\section{B. Singlet transitions}

The performance of the TD-DFT functionals were first tested on the main GSA band $\mathrm{S}_{0}\left(1^{1} \mathrm{~A}_{\mathrm{g}}\right) \rightarrow \mathrm{S}_{1}\left(1^{1} \mathrm{~B}_{\mathrm{u}}\right)$. BHLYP gives a vertical transition energy of $3.65 \mathrm{eV}$ (Table I), which compares reasonably well with the experimental band maximum (3.52 eV, Fig. 1), taking into account the missing bathochromic solvent shift in the calculation (amounting to ca. $-0.24 \mathrm{eV}){ }^{23}$ CAM-B3LYP performs similarly well (a) MOs

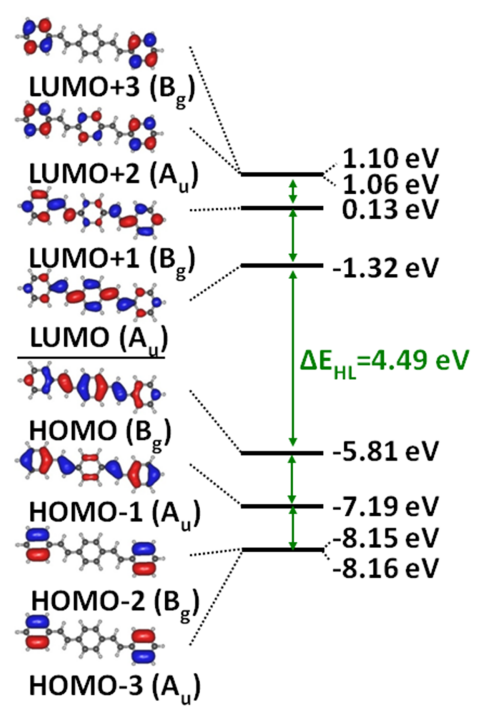

(b) Configurations (c) Singlet States

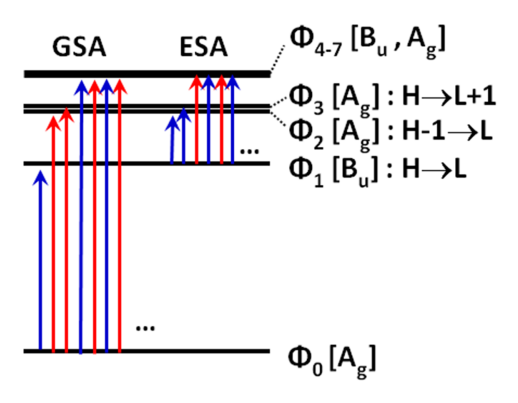

$\uparrow$ symmetry-allowed

$\uparrow$ symmetry-forbidden

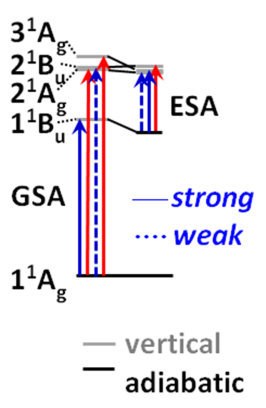

FIG. 2. Electronic and optical properties of DSB according to DFT calculations (BHLYP). (a) Energies and topologies (isosurface value 0.03 a.u.) of the four highest (lowest) (un)occupied MOs. (b) Configuration diagram of the allowed (blue arrows) and forbidden (red arrows) one-electron excitations. (c) Resulting singlet state diagram with vertical (grey) and adiabatic (black) transitions. 
TABLE I. Most prominent absorption bands in the GSA $\left(\mathrm{A}_{1}\right)$ and ESA spectra $\left(\mathrm{PA}_{\mathrm{i}}\right)$ : experimental band maxima $\mathrm{E}_{\text {max,exp }}$, band assignment, calculated vertical transition energies $\mathrm{E}_{\mathrm{vert}}$ (BHLYP), oscillator strength $f$, and CI description (coefficients and relative weights are in parentheses ).

\begin{tabular}{|c|c|c|c|c|c|}
\hline & $\mathrm{E}_{\max , \exp }(\mathrm{eV})$ & Assignment & $\mathrm{E}_{\mathrm{vert}}(\mathrm{eV})$ & $f$ & CI description \\
\hline $\mathrm{A}_{1}$ & 3.52 & $\mathrm{~S}_{0}\left(1^{1} \mathrm{~A}_{\mathrm{g}}\right) \rightarrow \mathrm{S}_{1}\left(1^{1} \mathrm{~B}_{\mathrm{u}}\right)$ & 3.65 & 1.92 & $\mathrm{H} \rightarrow \mathrm{L}(0.69,96 \%)^{\mathrm{a}}$ \\
\hline $\mathrm{PA}_{1}$ & 1.67 & $\mathrm{~S}_{1}\left(1^{1} \mathrm{~B}_{\mathrm{u}}\right) \rightarrow \mathrm{S}_{3}\left(3^{1} \mathrm{~A}_{\mathrm{g}}\right)$ & 1.45 & 0.63 & $\begin{array}{l}\mathrm{H} \rightarrow \mathrm{L}+1(-0.59,71 \%) \\
\mathrm{H}-1 \rightarrow \mathrm{L}(0.36,26 \%)\end{array}$ \\
\hline $\mathrm{PA}_{2}$ & $2.54^{\mathrm{b}}$ & $\mathrm{T}_{1}\left(1^{3} \mathrm{~B}_{\mathrm{u}}\right) \rightarrow \mathrm{T}_{4}\left(2^{3} \mathrm{~A}_{\mathrm{g}}\right)$ & 2.85 & 2.37 & $\begin{array}{l}\mathrm{H}(\alpha) \rightarrow \mathrm{L}(\alpha)(0.71,51 \%) \\
\mathrm{H}(\beta) \rightarrow \mathrm{L}(\beta)(-0.68,47 \%)\end{array}$ \\
\hline $\mathrm{PA}_{3}$ & $2.08^{\mathrm{b}}$ & $\mathrm{D}_{0}\left(1^{2} \mathrm{~B}_{\mathrm{g}}\right) \rightarrow \mathrm{D}_{2}\left(2^{2} \mathrm{~B}_{\mathrm{u}}\right)$ & 2.33 & 1.36 & $\begin{array}{l}\mathrm{H}-1(\alpha) \rightarrow \mathrm{H}(\alpha)(0.84,76 \%) \\
\mathrm{H}-1(\alpha) \rightarrow \mathrm{L}+1(\alpha)(-0.29,9 \%) \\
\mathrm{H}(\beta) \rightarrow \mathrm{L}(\beta)(0.25,6 \%)\end{array}$ \\
\hline
\end{tabular}

${ }^{\mathrm{a}} \mathrm{H}=\mathrm{HOMO}, \mathrm{L}=\mathrm{LUMO}$; in $\mathrm{D}_{0}$, the HOMO is only singly occupied.

${ }^{\mathrm{b}}$ Ref. 25 .

(3.67 eV), whereas B3LYP somewhat underestimates the band $(3.18 \mathrm{eV})$, see the supplementary material.

The main band in the experimental ESA spectrum at short probe delay times $\left(\mathrm{PA}_{1}\right)$ is centered at $1.67 \mathrm{eV}$ (see Fig. 1), followed by a small broad shoulder at around $1.8 \mathrm{eV}$, similar to what was reported earlier. ${ }^{24}$ Due to the agreement of the $\mathrm{PA}_{1}$ dynamics with the measured PL lifetime in solution, ${ }^{23}$ the band should originate from a S-S transition. At the BHLYP level, the band is assigned to $\mathrm{S}_{1}\left(1^{1} \mathrm{~B}_{\mathrm{u}}\right) \rightarrow \mathrm{S}_{3}\left(3^{1} \mathrm{~A}_{\mathrm{g}}\right)$, calculated at $\mathrm{E}_{\text {vert }}=1.45 \mathrm{eV}(f=0.63)$, see Table I. This is indeed the only strong singlet ESA in agreement with our simple configuration-based considerations made above. At the CAMB3LYP level, the main band is found at $1.51 \mathrm{eV}(f=0.80)$, while B3LYP gives only $0.68 \mathrm{eV}$ and $f=0.28$ (it should be noted that the CI descriptions for the latter is quite different from BHLYP; see Table I and the supplementary material). The underestimation of ESA transitions by B3LYP agrees with a former study on oligofluorenes; ${ }^{18}$ here, a possible contribution might arise from the missing double excitations in the TD-DFT scheme.

From the PPS spectrum at fast delay times in Fig. 1, we can calculate the experimental ratio of the oscillator strengths of the $\mathrm{PA}_{1}$ and the stimulated emission (SE) bands by comparing the integral spectral weights of these bands. We find a ratio $f\left(\mathrm{PA}_{1}\right) / f(\mathrm{SE})_{\exp }=1.0 \pm 0.2$, while the corresponding ratio in Table $\mathrm{I}$ is $\mathrm{r}_{\mathrm{BHLYP}}=f\left(\mathrm{PA}_{1}\right) / f\left(\mathrm{~A}_{1}\right)=0.33$. This number depends on the employed functional $\left(\mathrm{r}_{\mathrm{B} 3 \mathrm{LYP}}=0.15, \mathrm{r}_{\mathrm{CAM}-\mathrm{B} 3 \mathrm{LYP}}\right.$ $=0.43$ ); however, there is a general tendency to underestimate the strength of the $\mathrm{PA}_{1}$ band for all functionals. We highlight that in the experiment, the integral $f\left(\mathrm{~S}_{1} \rightarrow \mathrm{S}_{0}\right)$ can be underestimated by possible superposition with higher energetic excited state absorptions, not accounted for in the simulations; however, these possible contributions cannot account for a factor of three as required to match the data in Table I. Moreover, our data show that SE (caused by the $S_{1} \rightarrow S_{0}$ transition) and ground state bleach (GB, caused by the $S_{0} \rightarrow S_{1}$ transition) have approximately the same integral spectral weight, which speaks against a strong superposition with high energetic ESA bands in this spectral region. The underestimation of the $\mathrm{PA}_{1}$ intensity by the tested functionals exhibits a systematic correlation with the underestimation of its energy (B3LYP >> BHLYP > CAM-B3LYP; vide supra), and limits the application of these functionals for the quantitative prediction of
ESA spectra. For qualitative predictions however, the performance of BHLYP and CAM-B3LYP might be sufficient (as already remarked for the calculation of TPA spectra), ${ }^{21,22}$ in particular keeping in mind the simple structure of the spectrum with one intense peak.

A remarkable feature of $\mathrm{PA}_{1}$ in the ESA spectrum is the small bandwidth compared with $A_{1}$ in the GSA spectrum (see Fig. 1). This suggests very different geometrical reorganization upon electronic excitation, which can be obtained from the vertical and adiabatic transition energies by $E_{r e}=E_{\text {vert }}$ - $E_{a d}$. For $A_{1}$ in the GSA spectrum, the experimental $E_{r e}$ is $0.26 \mathrm{eV} ;{ }^{30}$ the calculated value (BHLYP) gives a similar result with $0.31 \mathrm{eV}$. The substantial $\mathrm{E}_{\mathrm{re}}$ upon $\mathrm{S}_{0} \rightarrow \mathrm{S}_{1}$ excitation is particularly seen in the vinylene unit by a lengthening (shortening) of the double (single) bond (Table II) due to the pronounced change in the electronic structure when going from the bonding $\pi$-type HOMO to the $\pi^{*}$-type LUMO, see Fig. 2. As shown earlier, ${ }^{30,44}$ for the same reason, the room temperature PL spectrum $\left(1^{1} \mathrm{~B}_{\mathrm{u}} \rightarrow 1^{1} \mathrm{~A}_{\mathrm{g}}\right)$ is better structured than the GSA spectrum (Fig. 1), since the shortening of the single bonds in $\mathrm{S}_{1}$ steepens the torsional potential around these bonds compared to $\mathrm{S}_{0}$; upon thermal population, this asymmetrically broadens the GSA, which can be described by an exponential convolution. ${ }^{30}$ Taking this into account, the BHLYP calculated PL and GSA vibronic spectra (Fig. 3) give indeed a reasonable agreement with the experiment (Fig. 1), similar to earlier HF CIS calculations. ${ }^{30}$

The geometrical reorganization for $\mathrm{PA}_{1}$ was calculated to $E_{\mathrm{re}}=0.02 \mathrm{eV}$ (BHLYP), indeed much smaller than in $A_{1}$. This reflects the small changes of the bond lengths in

TABLE II. Bond length in relevant electronic states (BHLYP): inner and outer phenyl-vinyl bonds $r_{i}, r_{o}$; vinyl double bond $r_{D B}$, see Fig. 1 .

\begin{tabular}{lccc}
\hline \hline & $\mathrm{r}_{\mathrm{i}}(\AA)$ & $\mathrm{r}_{\mathrm{o}}(\AA)$ & $\mathrm{r}_{\mathrm{DB}}(\AA)$ \\
\hline $\mathrm{S}_{0}\left(1^{1} \mathrm{~A}_{\mathrm{g}}\right)$ & 1.46 & 1.46 & 1.33 \\
$\mathrm{~S}_{1}\left(1^{1} \mathrm{~B}_{\mathrm{u}}\right)$ & 1.41 & 1.43 & 1.37 \\
$\mathrm{~S}_{3}\left(3^{1} \mathrm{~A}_{\mathrm{g}}\right)$ & 1.41 & 1.41 & 1.38 \\
$\mathrm{~T}_{1}\left(1^{3} \mathrm{~B}_{\mathrm{u}}\right)$ & 1.42 & 1.40 & 1.43 \\
$\mathrm{~T}_{4}\left(2^{3} \mathrm{~A}_{\mathrm{g}}\right)$ & 1.41 & 1.41 & 1.39 \\
$\mathrm{D}_{0}\left(1^{2} \mathrm{~B}_{\mathrm{g}}\right)$ & 1.43 & 1.44 & 1.37 \\
$\mathrm{D}_{2}\left(1^{2} \mathrm{~A}_{\mathrm{u}}\right)$ & 1.40 & 1.41 & 1.40 \\
\hline \hline
\end{tabular}




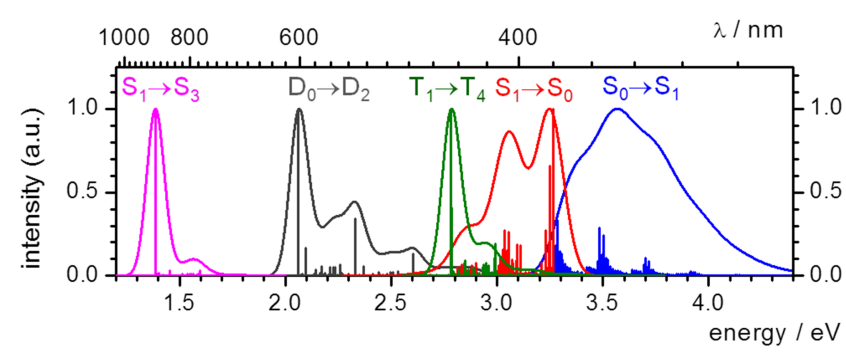

FIG. 3. DFT-calculated GSA and ESA full vibronic spectra of DSB (BHLYP): stick spectra and convoluted spectra (Gaussian half-width $0.1 \mathrm{eV}$; to account for solvent effects); the GSA spectrum was additionally convoluted with an exponential function to account for the thermal population of low frequency out-of-plane modes (see Sec. II). ${ }^{30}$

the vinylene units when going from $S_{1}$ to $S_{3}$ (Table II); the resulting potential curves of the singlet states projected along this coordinate are found in the supplementary material. Also for $\mathrm{PA}_{1}$, we calculated the full coupling of all vibrational modes to the electronic transition, see Fig. 3. Besides the apparent " $0-0$ " band at $1.38 \mathrm{eV}$, a small vibronic sideband at $1.59 \mathrm{eV}$ (" $0-1$ ") is revealed with an effective HuangRhys factor of $S=I_{0-1} / I_{0-0}=0.031$. This is very similar to the appearance of the experimental spectrum in Fig. 1. We thus assign the shoulder in the latter (i.e., at around $1.8 \mathrm{eV}$ ) mainly to the apparent $0-1$ subband of $S_{1} \rightarrow S_{3}$; it should be however noted that a higher electronic state of low intensity was calculated in this region $\left(\mathrm{S}_{1} \rightarrow \mathrm{S}_{6}\right.$ with $\mathrm{E}_{\mathrm{vert}}=1.88 \mathrm{eV}$, $f=0.04, \mathrm{E}_{\mathrm{re}}=0.15 \mathrm{eV}$; for details on the $\mathrm{S}_{\mathrm{n}}$ manifold see the supplementary material) which might equally contribute here.

\section{Triplet and polaronic transitions}

At long probe delay times, several features are visible in experiment. Most prominent is the band at $2.54 \mathrm{eV}\left(\mathrm{PA}_{2}\right.$; see Fig. 1). Following the most probable assignment by sensitization experiments, ${ }^{25}$ we calculated the T-T transitions by BHLYP, which resulted in only one band with considerable oscillator strength $(f=2.37)$, i.e. $\mathrm{T}_{1}\left(1^{3} \mathrm{~B}_{\mathrm{u}}\right) \rightarrow \mathrm{T}_{4}\left(2^{3} \mathrm{~A}_{\mathrm{g}}\right)$ at $2.85 \mathrm{eV}$ (Table I and the supplementary material), reproducing the experimental band position in an acceptable way; for CAM-B3LYP, $2.80 \mathrm{eV}$ is obtained, whereas for B3LYP, the transition is found at $2.10 \mathrm{eV}$. One of the reasons for the deviation from the experiment might be due to spin contamination in the calculations (see the supplementary material), being a rather common effect in triplet calculations with DFT. $^{45,46}$

The pronounced, structured background of the ESA spectrum cannot originate from further allowed T-T transitions, since no other transitions with significant oscillator strength were predicted. In the higher energy part, the features at $2.65 \mathrm{eV}$ in the experimental spectrum might be partially due to vibronics. Indeed, the calculated full vibronic spectrum of $\mathrm{PA}_{2}\left(\mathrm{~T}_{1} \rightarrow \mathrm{T}_{4}\right)$ in Fig. 3 reveals clear sidebands, quite similar to the experiment (Fig. 1).

We further calculated the absorption of the radical cations, following the tentative assignment in Ref. 25 for the peak at $2.08 \mathrm{eV}$ ( $\mathrm{PA}_{3}$; Fig. 1) based on the oxygen effect. Indeed, BHLYP gives an intense transition in this spectral region, i.e., $\mathrm{D}_{0}\left(1^{2} \mathrm{~B}_{\mathrm{g}}\right) \rightarrow \mathrm{D}_{2}\left(2^{2} \mathrm{~B}_{\mathrm{u}}\right)$ at $\mathrm{E}_{\mathrm{vert}}=2.33 \mathrm{eV}(f=1.36)$; the
$\mathrm{D}_{0} \rightarrow \mathrm{D}_{1}$ transition on the other side, calculated at $1.45 \mathrm{eV}$ and $f=0.61$ (see the supplementary material) is apparently outside the experimentally accessible range. It is worth pointing out that the spin contamination is not negligible here as well, see the supplementary material. Compared with the T-T absorption, the D-D absorption is weak in the experimental ESA spectrum. This indicates that the radical cation is a minor species (as expected); its relative concentration can be estimated from the absorbances $E$ of the $\mathrm{PA}_{i}$ bands $(i=2,3)$ as $\mathrm{x}$ $=E_{3} \cdot\left(f_{2}+f_{3}\right) /\left(\left(E_{2}+E_{3}\right) \cdot f_{3}\right) \approx 0.4$. The calculated pronounced vibronic sidebands (Fig. 3) are seen as the major source for the broad background in the $2.2-2.5 \mathrm{eV}$ range of the experimental spectrum; thus, in all, the ESA spectrum at long delay times is indeed mainly composed of one T-T and one D-D transition.

\section{DSB aggregates}

Based on the reasonable computational results for the ESA spectra of DSB in solution, a qualitative computational approach to the ESA spectra in the solid state was envisaged. It was shown in the past, that in single crystals ${ }^{41,47}$ as well as in nanoparticles (NP) or vapor-deposited films, ${ }^{23}$ nearest neighbors DSB molecules arrange side-by-side with their long axes, whereas the short axes are inclined, giving an edge-to-face (herringbone) assembly (see inset of Fig. 4) with $\mathrm{H}$-aggregation excitonic features of the GSA spectrum..$^{23,48}$ It is well-known from literature that the theoretical description of GSA features of molecular excitons in the medium coupling regime, as it is the case for DSB, requires the consideration of long-range interactions to properly reproduce the experimentally observed exciton couplings. ${ }^{49}$ Large cluster calculations are however not feasible for ESA spectra at the moment due to the limitations of the program. In any case, qualitative issues, in particularly on the question of the number of intense absorption bands are expected to be properly answered. In experiment, the GSA spectrum of small NPs of DSB reveals one intense peak in the near UV at $4.20 \mathrm{eV}$, hypsochromically shifted by $0.7 \mathrm{eV}$ against solution, ${ }^{23}$ which is assigned to the upper exciton band edge (H-band) ${ }^{48}$ whereas at the red side of the spectrum, the lowest visible feature is found at $3.12 \mathrm{eV}^{23}$ In the ESA spectrum at short delay times, one prominent band is observed at around $1.6 \mathrm{eV},{ }^{50}$ very similar to the result in the dilute solution.

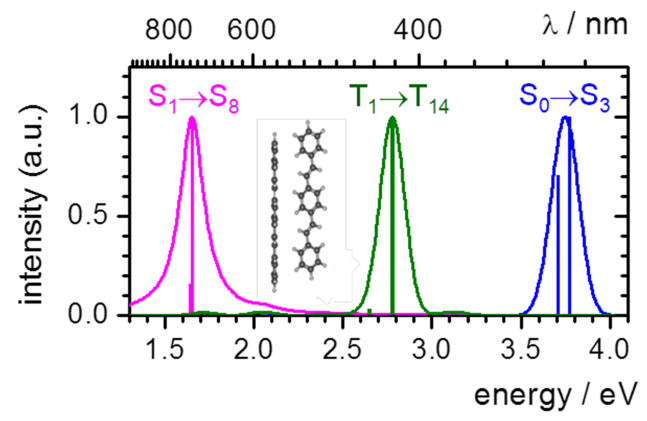

FIG. 4. Main GSA and ESA vertical transitions for a dimer of DSB as calculated at the DFT (BHLYP) level (Gaussian half-width of $0.25 \mathrm{eV}$ ); the inset shows the nearest neighbor arrangement as obtained from the single crystal $\mathrm{X}$-ray analysis. 
Vertical transitions of the GSA spectrum were calculated for a dimer and tetramer arrangement. The most intense transition of the dimer $\left(\mathrm{S}_{0} \rightarrow \mathrm{S}_{3}\right)$ is calculated at the BHLYP level to $3.80 \mathrm{eV}$ (CAMB3LYP: $3.75 \mathrm{eV} ; \mathrm{S}_{0} \rightarrow \mathrm{S}_{2}$, and wB97XD; ${ }^{51}$ $3.82 \mathrm{eV} ; \mathrm{S}_{0} \rightarrow \mathrm{S}_{2}$ see the supplementary material), and for the tetramer, $3.85 \mathrm{eV}\left(\mathrm{S}_{0} \rightarrow \mathrm{S}_{4}\right)$ is obtained. The transition reveals significant charge transfer (CT) contributions, see the supplementary material. The spectral shift against the single molecule (3.65 eV; Table I) is significantly smaller than in experiment; this might be partially due to the missing solvent effect in the single molecule calculation, but also due to the missing long-range interactions and polarizability effects in the small clusters. In any case, the size consistency seems to be sufficient to make qualitative statements for the following ESA spectra based on the dimer calculations. The calculated S-S ESA spectrum is dominated by a single peak at $1.65 \mathrm{eV}\left(\mathrm{S}_{1} \rightarrow \mathrm{S}_{8}\right)$ very similar to the experimental spectrum, where the similarity of the energetic position with the isolated molecule can be traced back to the localized character of the excitation giving essentially the same electronic transition as in the monomer. In the triplet ESA spectrum, the most intense peak is calculated at $2.77 \mathrm{eV}\left(\mathrm{T}_{1} \rightarrow \mathrm{T}_{14}\right)$, slightly redshifted to what is observed in our results for a single molecule $(2.84 \mathrm{eV})$.

\section{CONCLUSIONS}

Ground and excited state absorption spectra (GSA, ESA) were calculated by TD-DFT for DSB, being an experimentally well studied model compound for organic optoelectronics. The principle features of the singlet GSA and ESA spectra with one strong dominating peak can be understood on the basis of a simple $\mathrm{MO}$ and configuration picture. However, while the main GSA is shown to be essentially a monoelectronic excitation (HOMO $\rightarrow$ LUMO), the ESA features suffer from strong CI, making TD-DFT calculations more vulnerable against variations in the functional. Within the rather limited range of functionals available for the ESA calculations, B3LYP could not be recommended according to the present study, while BHLYP and CAM-B3LYP performed qualitatively well; quantitatively, however, the performance of both functionals (in terms of energies and oscillator strengths of singlet and triplet ESA transitions) clearly calls for further improvements. Our full vibronic calculations revealed the essential details of the ESA spectra of DSB in solution at short and long probe delay times, assigning the S-S, T-T, and $\mathrm{D}-\mathrm{D}$ transitions, elucidating the origin of strongly varying bandwidths, and proving the vibronic nature of the sidebands observed. Finally, a qualitative approach to thin film spectra gave a reasonable agreement with the experiment, stressing the need for supramolecular approaches for the calculation of solid state ESA for systems in the medium exciton coupling regime.

The results presented herein can be qualitatively extended to other conjugated systems, in particular to polymers by extrapolation. In poly-3-hexylthiophene (P3HT) for instance, the simple structure of ESA spectra is preserved, as shown in the paper just published during the revision of the current manuscript. ${ }^{52}$ In any case, our own (unpublished) calculations on P3HT reveal an increasingly complex CI description with longer chain length due to smaller energetic separations in the MO manifold with longer chain length. Moreover, the error as observed here for DSB is becoming more sincere similar to the case of GSA energies, ${ }^{13,16}$ due to the known inherent overestimation of delocalization in DFT functionals with low to medium Hartree-Fock exchange. Further steps ahead are a proper inclusion of double excitations for mediumand large-sized molecules, as well as the lateral extension of molecular assemblies to correctly account for long-range interactions.

\section{SUPPLEMENTARY MATERIAL}

See supplementary material for more details on the GSA and ESA computational results.

\section{ACKNOWLEDGMENTS}

We would like to thank the Brazilian agency FAPESP (Proc. Nos. 2012/21983-0, 2014/20410-1, and 2016/012886) for financial support. This research was also supported by resources supplied by the Center for Scientific Computing (NCC/GridUNESP) of the São Paulo State University (UNESP). The work at IMDEA and Univ. Val. was supported by the Spanish Ministerio de Economá y Competitividad (MINECO-FEDER Project No. CTQ2014-58801); the work at IMDEA further by the Comunidad de Madrid (Project PhotoCarbon No. S2013/MIT-284) and by the Campus of International Excellence (CEI) UAM+CSIC. We further thank the EC for access to the facility "Laserlab-Europe" (FP7Infrastructures-2008-1; Contract No. 228334 CUSBO). L.L. acknowledges funding from the EC via the COFUND program AMAROUT. J.S. acknowledges a Ph.D. grant of the Chinese Scholarship Council (CSC).

${ }^{1}$ W. Brütting and C. Adachi, Physics of Organic Semiconductor, 2nd ed. (Wiley-VHC, Weinheim, 2012).

${ }^{2}$ D. L. Andrews and A. A. Demidov, An Introduction to Laser Spectroscopy, 2nd ed. (Springer, New York, 2002).

${ }^{3}$ M. Fushitani, Annu. Rep., Sect. C: Phys. Chem. 104, 272 (2008).

${ }^{4}$ J. Cabanillas-Gonzales, G. Grancini, and G. Lanzani, Adv. Mater. 23, 5468 (2011).

${ }^{5}$ I. H. Van Stokkum, D. S. Larsen, and R. van Grondelle, Biochim. Biophys. Acta, Bioenerg. 1657, 82 (2004).

${ }^{6}$ C. Dyer-Smith, I. A. Howard, C. Cabanetos, A. E. Labban, P. M. Beaujuge, and F. Laquai, Adv. Energy Mater. 5, 1401778 (2015).

${ }^{7}$ L. Lüer, A.-M. Carey, S. Henry, M. Maiuri, K. Hacking, D. Polli, G. Cerullo, and R. J. Cogdell, Biophys. J. 109, 1885 (2015).

${ }^{8}$ Z. Yu, X. Guo, Q. Zhang, L. Chi, T. Chen, R. Xia, L. Wu, L. Lüer, and J. Cabanillas-Gonzalez, J. Phys. Chem. C 120, 11350 (2016).

${ }^{9}$ S. Karuthedath, T. Sauermann, H.-J. Egelhaaf, R. Wannemacher, C. J. Brabec, and L. Lüer, J. Mater. Chem. A 3, 3399 (2015).

${ }^{10}$ I. F. Domínguez, A. Distler, and L. Lüer, Adv. Energy Mater. 7, 1601320 (2017).

${ }^{11}$ J. R. Ochsmann, D. Chandran, D. W. Gehrig, H. Anwar, P. K. Madathil, K.-S. Lee, and F. Laquai, Macromol. Rapid Commun. 36, 1122 (2015).

${ }^{12}$ P. J. Brown, H. Sirringhaus, M. Harrison, M. Shkunov, and R. H. Friend, Phys. Rev. B 62, 125204 (2001).

${ }^{13}$ M. Wykes, B. Milián-Medina, and J. Gierschner, Front. Chem. 1, 1 (2013).

${ }^{14}$ B. Milián-Medina and J. Gierschner, Wiley Interdiscip. Rev.: Comput. Mol. Sci. 2, 513 (2012).

${ }^{15}$ A. Jain, Y. Shin, and K. A. Persson, Nat. Rev. Mater. 1, 15004 (2016).

${ }^{16}$ E. F. Oliveira, J. C. Roldao, B. Milián-Medina, F. C. Lavarda, and J. Gierschner, Chem. Phys. Lett. 645, 169 (2016). 
${ }^{17}$ L. González, D. Escudero, and L. Serrano-Andrés, Chem. Phys. Chem. 13, 28 (2012).

${ }^{18}$ S. Ling, S. Schumacher, I. Galbraith, and M. J. Paterson, J. Phys. Chem. C 117, 6889 (2013).

${ }^{19}$ J.-C. Denis, A. Ruseckas, G. J. Hedley, A. B. Matheson, M. J. Paterson, G. A. Turnbull, I. D. W. Samuel, and I. Galbraith, Phys. Chem. Chem. Phys. 18, 21937 (2016).

${ }^{20}$ A. Masunov and S. Tretiak, J. Phys. Chem. B 108, 899 (2004).

${ }^{21}$ M. T. P. Beerepoot, D. H. Friese, N. H. List, J. Kongsted, and K. Ruud, Phys. Chem. Chem. Phys. 17, 19306 (2015).

${ }^{22}$ I. H. Nayyar, A. E. Masunov, and S. Tretiak, J. Phys. Chem. C 117, 18170 (2013).

${ }^{23}$ J. Gierschner, L. Lüer, B. Milián-Medina, D. Oelkrug, and H.-J. Egelhaaf, J. Phys. Chem. Lett. 4, 2686 (2013).

${ }^{24}$ G. Ginocchietti, E. Cecchetto, L. De Cola, U. Mazzucato, and A. Spalletti, Chem. Phys. 352, 28 (2008).

${ }^{25}$ E. Marri, F. Elisei, U. Mazzucato, D. Pannacci, and A. Spalletti, J. Photochem. Photobiol. A: Chem. 177, 307 (2006).

${ }^{26}$ G. Cerullo, C. Manzoni, L. Luer, and D. Polli, Photochem. Photobiol. Sci. 6, 135 (2007).

${ }^{27}$ N. F. Colaneri, D. D. C. Bradley, R. H. Friend, P. L. Burn, A. B. Holmes, and C. W. Spangler, Phys. Rev. B 42, 11670 (1990).

${ }^{28}$ J. Gierschner, M. Ehni, H.-J. Egelhaaf, B. Milián-Medina, D. Beljonne, H. Benmansour, and G. C. Bazan, J. Chem. Phys. 123, 144914 (2005).

${ }^{29}$ J. Gierschner, J. Cornil, and H.-J. Egelhaaf, Adv. Mater. 19, 173 (2007).

${ }^{30}$ J. Gierschner, H.-G. Mack, L. Lüer, and D. Oelkrug, J. Chem. Phys. 116, 8596 (2002).

${ }^{31}$ M. Wykes, R. Parambil, D. Beljonne, and J. Gierschner, J. Chem. Phys. 143, 114116 (2015).

${ }^{32}$ M. A. L. Marques, N. T. Maitra, F. M. S. Nogueira, E. K. U. Gross, and A. Rubio, Fundamentals of Time-Dependent Density Functional Theory, 1st ed. (Springer-Verlag, Berlin, 2012).

${ }^{33}$ M. J. Frisch, G. W. Trucks, H. B. Schlegel, G. E. Scuseria, M. A. Robb, J. R. Cheeseman, G. Scalmani, V. Barone, B. Mennucci, G. A. Petersson, H. Nakatsuji, M. Caricato, X. Li, H. P. Hratchian, A. F. Izmaylov, J. Bloino, G. Zheng, J. L. Sonnenberg, M. Hada, M. Ehara, K. Toyota, R. Fukuda, J. Hasegawa, M. Ishida, T. Nakajima, Y. Honda, O. Kitao, H. Nakai, T. Vreven, J. A. Montgomery, Jr., J. E. Peralta, F. Ogliaro, M. Bearpark, J. J. Heyd, E. Brothers, K. N. Kudin, V. N. Staroverov, R. Kobayashi, J. Normand, K. Raghavachari, A. Rendell, J. C. Burant, S. S. Iyengar, J. Tomasi, M. Cossi, N. Rega, J. M. Millam, M. Klene, J. E. Knox, J. B. Cross, V. Bakken, C. Adamo, J. Jaramillo, R. Gomperts, R. E. Stratmann, O. Yazyev, A. J. Austin, R. Cammi, C. Pomelli, J. W. Ochterski, R. L. Martin, K. Morokuma, V. G. Zakrzewski, G. A. Voth, P. Salvador, J. J. Dannenberg, S. Dapprich, A. D. Daniels, Ö. Farkas, J. B. Foresman, J. V. Ortiz, J. Cioslowski, and D. J. Fox, Gaussian 09, Revision D.01, Gaussian, Inc., Wallingford, CT, 2009.

${ }^{34}$ K. Aidas, C. Angeli, K. L. Bak, V. Bakken, R. Bast, L. Boman, O. Christiansen, R. Cimiraglia, S. Coriani, P. Dahle, E. K. Dalskov,
U. Ekström, T. Enevoldsen, J. J. Eriksen, P. Ettenhuber, B. Fernández, L. Ferrighi, H. Fliegl, L. Frediani, K. Hald, A. Halkier, C. Hättig, H. Heiberg, T. Helgaker, A. C. Hennum, H. Hettema, E. Hjertenæs, S. Høst, I.-M. Høyvik, M. F. Iozzi, B. Jansík, H. J. A. Jensen, D. Jonsson, P. Jørgensen, J. Kauczor, S. Kirpekar, T. Kjærgaard, W. Klopper, S. Knecht, R. Kobayashi, H. Koch, J. Kongsted, A. Krapp, K. Kristensen, A. Ligabue, O. B. Lutnæs, J. I. Melo, K. V. Mikkelsen, R. H. Myhre, C. Neiss, C. B. Nielsen, P. Norman, J. Olsen, J. M. H. Olsen, A. Osted, M. J. Packer, F. Pawlowski, T. B. Pedersen, P. F. Provasi, S. Reine, Z. Rinkevicius, T. A. Ruden, K. Ruud, V. V. Rybkin, P. Sałek, C. C. M. Samson, A. S. de Merás, T. Saue, S. P. A. Sauer, B. Schimmelpfennig, K. Sneskov, A. H. Steindal, K. O. Sylvester-Hvid, P. R. Taylor, A. M. Teale, E. I. Tellgren, D. P. Tew, A. J. Thorvaldsen, L. Thøgersen, O. Vahtras, M. A. Watson, D. J. D. Wilson, M. Ziolkowski, and H. Ågren, Wiley Interdiscip. Rev.: Comput. Mol. Sci. 4, 269 (2014).

${ }^{35}$ A. D. Becke, J. Chem. Phys. 98, 1372 (1993).

${ }^{36}$ P. J. Stephens, F. J. Devlin, C. F. Chabalowski, and M. J. Frisch, J. Phys. Chem. 98, 11623 (1994).

${ }^{37}$ A. D. Becke, J. Chem. Phys. 98, 5648 (1993).

${ }^{38}$ Y. Zhao and D. G. Truhlar, J. Phys. Chem. A 110, 13126 (2006).

${ }^{39}$ J. Gierschner, H.-G. Mack, H.-J. Egelhaaf, S. Schweizer, B. Doser, and D. Oelkrug, Synth. Met. 138, 311 (2003).

${ }^{40}$ F. Santoro, FCClasses, a Fortran 77 code, visit http://www.pi.iccom. cnr.it/fcclasses.

${ }^{41}$ F. J. Avila Ferrer, J. Cerezo, J. Soto, R. Improta, and F. Santoro, Comput. Theor. Chem. 1040-1041, 328 (2014).

${ }^{42}$ S. Varghese, S. K. Park, S. Casado, R. Fischer, R. Resel, B. Milián-Medina, R. Wannemacher, S. Y. Park, and J. Gierschner, J. Phys. Chem. Lett. 4, 1597 (2013)

${ }^{43}$ D. Oelkrug, J. Gierschner, H.-J. Egelhaaf, L. Lüer, A. Tompert, K. Müllen, U. Stalmach, and H. Meier, Synth. Met. 121, 1693 (2001).

${ }^{44}$ G. Srinivasan, J. A. Villanueva-Garibay, K. Müller, D. Oelkrug, B. Milián-Medina, D. Beljonne, J. Cornil, M. Wykes, L. Viani, J. Gierschner, R. Martinez-Alvarez, M. Jazdzyk, M. Hanack, and H.-J. Egelhaaf, Phys. Chem. Chem. Phys. 11, 4996 (2009).

${ }^{45}$ M. J. G. Peach, M. J. Williamson, and D. J. Tozer, Mol. Phys. 111, 1271 (2013).

${ }^{46}$ J. S. Sears, T. Koerzdoerfer, C.-R. Zhang, and J.-L. Brédas, J. Chem. Phys. 135, 151103 (2011).

${ }^{47}$ C. C. Wu, M. C. DeLong, Z. V. Vardeny, J. P. Ferraris, and J. J. Gutierrez, Synth. Met. 137, 939 (2003).

${ }^{48}$ F. C. Spano, J. Chem. Phys. 118, 981 (2003).

${ }^{49}$ J. Gierschner, Y.-S. Huang, B. Van Averbeke, J. Cornil, R. H. Friend, and D. Beljonne, J. Chem. Phys. 130044105 (2009).

${ }^{50}$ G. Cerullo, G. Lanzani, S. De Silvestri, H.-J. Egelhaaf, L. Lüer, and D. Oelkrug, Phys. Rev. B 62, 2429 (2000).

${ }^{51}$ J.-D. Chai and M. Head-Gordon, Phys. Chem. Chem. Phys. 10, 6615 (2008).

${ }^{52}$ R. B. Roseli, C. Tapping, and T. W. Kee, J. Phys. Chem. Lett. 8, 2806 (2017). 